\title{
Six New and Four Unrecorded Species of Tanytarsini (Diptera, Chironomidae, Chironominae) Found in Korea
}

\author{
Han-Il Ree ${ }^{1,2, *}$, Kyoung Yong Jeong ${ }^{2,3}$, Sung Hyun Nam ${ }^{1,2}$ \\ ${ }^{1}$ Department of Environmental Medical Biology and Institute of Tropical Medicine, \\ College of Medicine, Yonsei University, Seoul 120-752, Korea \\ ${ }^{2}$ Arthropods of Medical Importance Resource Bank, College of Medicine, \\ Yonsei University, Seoul 120-752, Korea \\ ${ }^{3}$ Department of Internal Medicine and Institute of Allergy, College of Medicine, \\ Yonsei University, Seoul 120-752, Korea
}

\begin{abstract}
Adult chironomid collections were carried out near Namdae stream located at Jeollabuk-do, Muju-gun, Mujueup, Dangsan-ri in 2008 and 2009. Among 21 species of the tribe Tanytarsini identified from Muju collections, six new species (Cladotanytarsus neovanderwulpi, Paratanytarsus paramikesecumdus, Rheotanytarsus parapentapodus, Rheotanytarsus sungili, Tanytarsus neotamaoctavus, and Tanytarsus synyunosecundus) and four previously unrecorded species (Cladotanytarsus vanderwulpi, Paratanytarsus inopertus, Tanytarsus tamagotoi, and Tanytarsus uresiacutus) were confirmed. They are fully described with illustrations. As a result of this report, the Korean fauna of Tanytarsini consists of 37 species, 6 genera. In total, 128 species, 52 genera, 5 subfamilies of the family Chironomidae are listed in Korea.
\end{abstract}

Keywords: Chironomidae, new species, Tanytarsini, taxonomy

\section{INTRODUCTION}

Adults of non-biting midges (Diptera, Chironomidae) were collected at Jeollabuk-do, Muju-gun, Muju-eup, Dangsan-ri on 5 September 2008, 22 May 2009 and 28 August 2009. Collection sites were near Namdae-stream which runs through the western area of Muju-eup. Chironomid adults attracted to the light sources of buildings such as restaurants, stores, police substation and the health center were aspirated by a suckling tube, and preserved in $75 \%$ ethanol. Later, they were dissected under stereomicroscope using fine needles to retrieve antennae, head, wings and hypopygium, and were mounted on polyvinyl alcohol medium. They are presently fully described with illustrations. Chironominae is the largest subfamily among eight subfamilies of the family Chironomidae, and three tribes are recognized: Chironomini, Pseudochironomini and Tanytarsini. The tribe Tanytarsini is characterized by wing with macrotrichia, squama bare, RM parallel to $\mathrm{R}_{4+5}$, and hypopygium with median volsella. In Korea, two species of Tanytarsini was reported for the first time by Ree

(C) This is an Open Access article distributed under the terms of the Creative Commons Attribution Non-Commercial License (http://creativecommons.org/ licenses/by-nc/3.0/) which permits unrestricted non-commercial use, distribution, and reproduction in any medium, provided the original work is properly cited. and Kim (1988), one species by Ree (1989), two species by Ree (1992), one species by Ree and Kim (2003), four species by $\mathrm{Na}$ (2004), and seven species by Ree and Jeong (2010). Adding 10 species by the present paper, total 27 species of Tanytarsini are recorded. The type specimens have been deposited in the collection of Arthropods of Medical Importance Resource Bank, Department of Environmental Medical Biology, Yonsei University College of Medicine. Terminology follows that of Saether (1980).

\section{SYSTEMATIC ACCOUNTS}

Order Diptera

Family Chironomidae Haliday

Subfamily Chironominae Macquart

Tribe Tanytarsini

Genus Cladotanytarsus Kieffer, 1922

${ }^{1 *}$ Cladotanytarsus neovanderwulpi sp. nov.

Material examined. Holotype, ه (R-CH-6987): Korea:

\section{*To whom correspondence should be addressed \\ Tel: 82-2-2228-1840, Fax: 82-2-363-8676}

E-mail: para@yuhs.ac 
Jeollabuk-do, Muju-gun, Muju-eup, Dangsan-ri, 28 Aug 2009, Jeong KY. Paratypes, 1 శ (R-CH-6463), locality and collector same as holotype, 2 Sep 2008; 6 ठ す (R-CH-7057, 7072, 7076, 7117, 7128, 7149), same as holotype.

Diagnosis. Small, yellowish species, with dark brown scutal vittae and postnotum. Wing length (WL) $1.1 \mathrm{~mm}$. Eyes reniform. Wing membrane with macrotrichiae on distal end only. Superior volsella, slightly tapered apically, tip bent upward, with 3-4 dorsal setae and 3 basal setae arised from cylindrical tube; digitus long. Median volsella short, with branched setae. Antennal ratio (AR) 0.73. Leg ratio (LR) 2.27 .

Description (male). Head: Pale yellow. Eye bare, not dorsomedially produced. Frontal tubercle absent. Antenna pale dark brown, with 13 segments. AR 0.73. Clypeus pale yellow, with 9 setae. Palp pale, with 5 segments: 29, 32, 71, $125,143 \mu \mathrm{m}(1: 1.1: 2.5: 4.3: 4.9)$. Thorax: Pale yellow in ground color. Anteprotonum pale yellow, narrowed dorsally, separated medially. Scutum overreaching antepronotum, with dark brown vittae. Scutellum dark brown, with 2 scutellars. Postnotum dark brown. Preepisternum dark brown. Halter pale yellow. Wing(Fig. 1A): WL $1.1 \mathrm{~mm}$. Membrane with macrotrichiae only apically. $R_{2+3}$ ending in proximal $1 / 3$ between apices of $\mathrm{R}_{1}$ and $\mathrm{R}_{4+5}$. Only $\mathrm{R}_{4+5}$ with setae distally. $\mathrm{RM}$ proximal to $\mathrm{FCu}$. An scarcely reaching $\mathrm{FCu}$. Anal lobe not developed. Squama bare. Arculus dark brown. Brachiolum pale, bare. Legs: All legs pale (foreleg slightly darker). Tibiae of mid and hind legs with separated combs, each with a spur. Pulvillus absent. LR 2.27. Abdomen: Uniformly pale yellow. Hypopygium (Fig. 1B): Anal tergite not apically produced, with 7-9 minute median setae, close to spines of anal point; anal tergite band pale and widely separated. Anal point partly overlying anal tergite, dully pointed apically, with distinct crests and 6-9 irregularly directed spinules. Superior volsella slightly tapered apically, tip bent upward, with 3-4 dorsal setae and 3 basal setae arised from small cylindrical base; long digitus distinctly produced. Median volsella short, with branched lamellar setae. Inferior volsella cylindrical with 7-10 rather short setae, apical long seta absent. Gonostylus rather short, small, with smoothly rounded apex.

Etymology. This new species is named for Cl. vanderwulpi, very close relative species.

Distribution. Korea (Muju).

Remarks. This new species is very similar to Cladotanytarsus vanderwulpi morphologically, but differs in several key characters: (1) the median vosella is short, whereas that of C. vanderwulpi is very long and curved; (2) the anal point has 6-9 spine clusters, whereas later species has 4-5 spine clusters, and (3) the LR is 2.27, whereas that of $C$. vanderwulpi is 2.73 (Pinder, 1978; Sasa, 1985).

\section{1 *Cladotanytarsus vanderwulpi (Edwards, 1929)}

Tanytarsus (Cladotanytarsus) vanderwulpi Edwards, 1929: 418.

Tanytarsus unagisextus Sasa, 1985: 46.

Cladotanytarsus vanderwulpi: Pinder, 1978: 142; Sasa, 1985 : 44; Sasa and Kawai, 1987: 28.

Material examined. 14 ఠ gun, Muju-eup, Dangsan-ri, 5 Sep 2008, Jeong KY, Nam SH. Diagnosis. Small, pale yellow midge (WL $1.1 \mathrm{~mm}$ ). Eye bare, without dorsomedial extension. Wing membrane with macrotrichiae on distal 1/3. Anal point short, conical, pointed at tip, with anal crest and 4-5 spines. Superior volsella apically tapered, with 3-4 dorsal and 2 basal setae; digitus long, finger-like. Median volsella long, curved at middle, with branched setae. AR 0.74. LR 2.73.

Description (male). Head: Eye bare, not dorsomedially produced. Frontal tubercle absent. 10-11 postoculars each side. Antenna yellowish brown, with 13 segments. AR 0.74 . Clypeus pale yellow, with 13 setae. Palp pale yellow, with 5 segments: $25,25,86,93,136 \mu \mathrm{m}(1: 1: 3.4: 3.7: 5.4)$. Thorax: Yellowish in ground color. Antepronotum pale yellow, bare, smoothly tapered dorsally, well separated each other. Scutum pale yellow, with brownish yellow vittae, 6-7 dorsocentals each side. Scutellum pale yellow, with 4 setae. Postnotum brownish yellow. Halter pale. Wing (Fig. 2A): WL $1.1 \mathrm{~mm}$. Membrane with macrotrichiae on distal $1 / 3$ only. $\mathrm{R}, \mathrm{R}_{1}$, distal $1 / 2$ of $\mathrm{R}_{4+5}$, distal $1 / 2$ of $\mathrm{M}_{1+2}$ setosed. Costa not produced. $\mathrm{R}_{2+3}$ ending about on middle between ends of $\mathrm{R}_{1}$ and $\mathrm{R}_{4+5}$. $\mathrm{RM}$ well proximal to $\mathrm{FCu}$. $\mathrm{Cu}_{1}$ almost straight. An scarcely reaching FCu. Anal lobe not developed. Squama bare. Brachiolum pale, with 1 seta, arculus dark brown. Legs: all segments uniformly pale yellow. LR 2.73 . Abdomen: Uniformly pale yellow. Hypopygium (Fig. 2B): Anal tergite with 4-5 small medium setae, 2-3 small apical setae on each side of anal point, and weakly developed apodeme. Anal point short, conical, pointed at tip, with anal crest and 4-5 spine clusters. Superior volsella ovoid with short apical lobe, and 3-4 dorsal and 2 inner-basal setae; digitus long, narrow, finger-like. Median vosella long, curved at middle, with branched setae. Inferior volsella cylindrical with 8-11 rather short setae apically. Gonostylus rather short, smoothly rounded tip, with an apical and several subapical short setae.

Distribution. Europe (England), Mongolia, China, Japan, Korea.

Korean name: ${ }^{1 *}$ 울프눈장부깔따구 

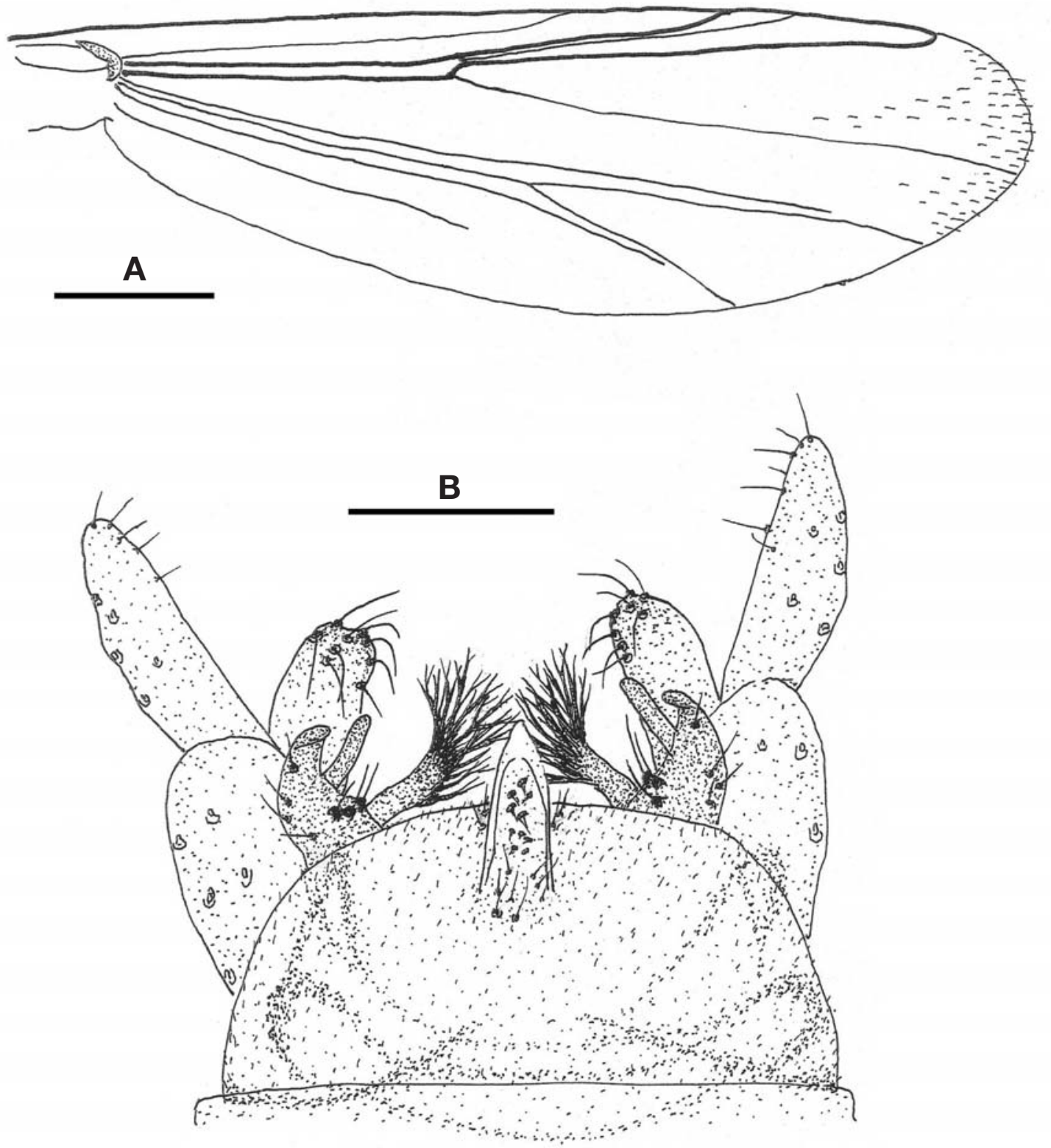

Fig. 1. Cladotanytarsus neovanderwulpi sp. nov. (male). A, Wing; $B$, Hypopygium. Scale bars: $A=0.2$ mm, $B=0.05$ mm.

Remarks. Cladotanytarsus vanderwulpi was recorded in England (Edwards, 1929), Japan (Sasa, 1985; Sasa and Kawai, 1987), China (Wang and Zheng, 1992) and Mongolia (Shcherbina and Zelentsov, 2008). Our specimens coincide in general with those specimens, except that wing length of the England specimens $(2.0 \mathrm{~mm}$ ) (Edwards, 1929) is larger than those of the Japanese (1.1-1.3 mm) (Sasa, 1985) and Korean specimens $(1.1 \mathrm{~mm})$.
Genus Paratanytarsus, Thienemann and Bause, 1913

\section{1*Paratanytarsus inopertus (Walker, 1856)}

Chironomus inopertus Walker, 1856: 164.

Tanytarsus inopertus: Edwards, 1929: 410; Goetghebuer, 1937: 117.

Paratanytarsus inopertus: Pinder, 1978: 148; Sasa and Kamimura, 1987: 22. 


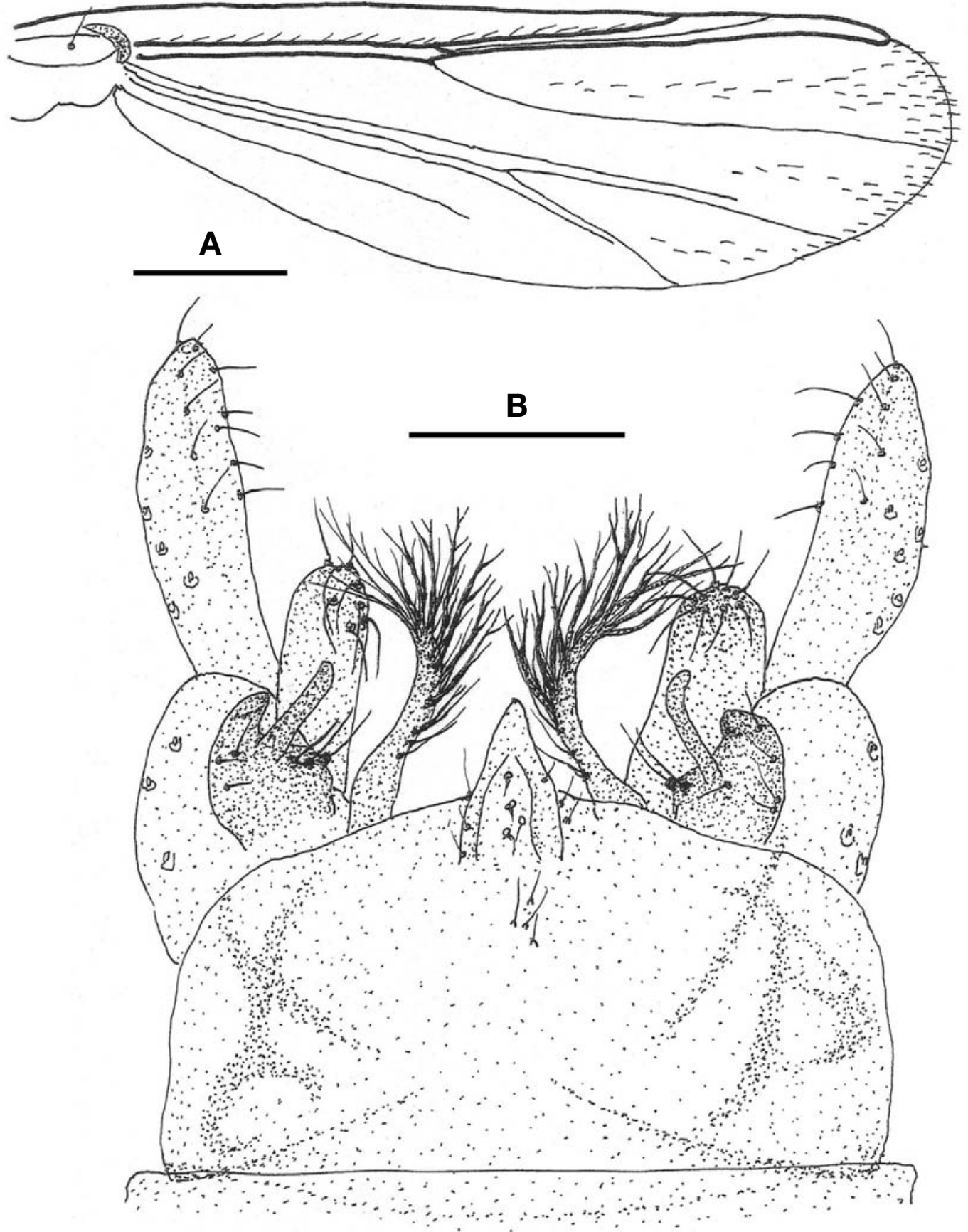

Fig. 2. Cladotanytarsus vanderwulpi (male). A, Wing; $B$, Hypopygium. Scale bars: $A=0.2 \mathrm{~mm}, B=0.05 \mathrm{~mm}$.

Material examined. $12 \diamond^{\nearrow} \sigma^{\nearrow, ~ K o r e a: ~ J e o l l a b u k-d o, ~ M u j u-~}$ gun, Muju-eup, Dangsan-ri, 22 May 2009, Jeong KY; $2 \sigma^{\nearrow} \sigma^{\nearrow}$, locality same, 28 Aug 2009, Jeong KY.

Diagnosis. Light brown, medium to small midge (WL 1.7 $\mathrm{mm}$ ). Wing membrane heavily covered with macrotrichiae. Superior volsella round, with 9-10 dorsal setae; digitus pointed at tip, slightly extending beyond margin of superior volsella. Median volsella moderately long, slightly curved at tip, with simple setae. Anal point short, flattened at tip, with unique anal crests. AR 1.22. LR 1.60.

Description (male). Head: Eye bare, with dorsomedial projection. Frontal tubercle present (18 $\mu \mathrm{m}$ long) (Fig. 3B). Antenna pale dark brown, with 13 segments, AR 1.22. Palp pale yellow, with 4 segments: $30,107,107,143 \mu \mathrm{m}$ (1 : 3.6 : 3.6 : 

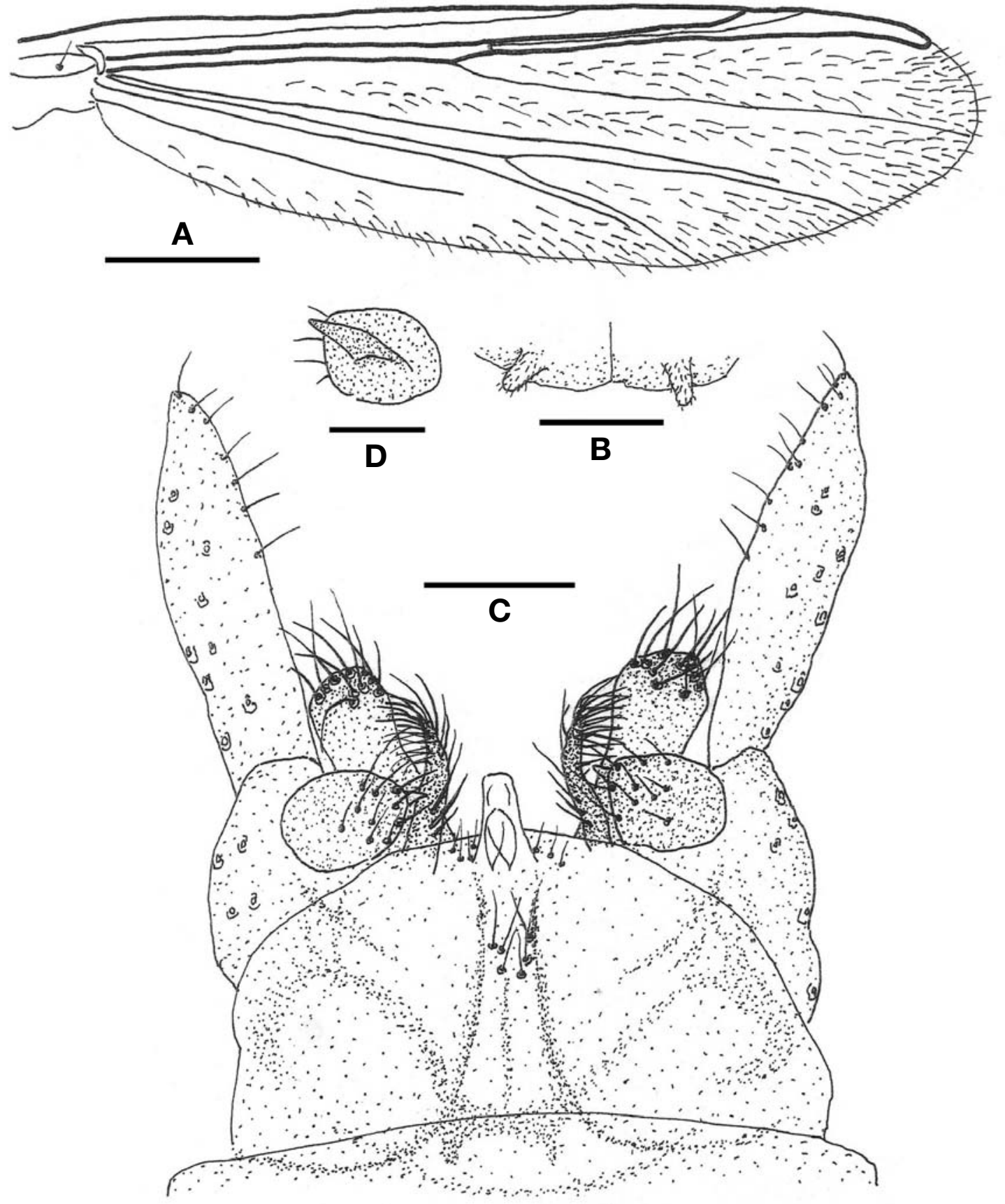

Fig. 3. Paratanytarsus inopertus (male). A, Wing; B, Frontal tubercle; C, Hypopygium; D, Superior vollsella (ventral). Scale bars: $A=0.3 \mathrm{~mm}, B, C=0.05 \mathrm{~mm}, D=0.03 \mathrm{~mm}$.

4.8). Clypeus pale yellow, with 15 setae. Thorax: Brownish yellow in ground color. Antepronotum brownish yellow, narrowed dorsally, widely separated each other. Scutum yellowish brown, remarkably overreaching antepronotum, vittae dark brown. Scutellum yellowish brown, with 9 setae. Postnotum dark brown. Prespisternum dark brown. Halter pale. Wing (Fig. 3A): WL $1.7 \mathrm{~mm}$. Membrane covered with macrotrichiae. Costa not produced. $R_{2+3}$ ending at $1 / 3$ basal between ends of $R_{1}$ and $R_{4+5}$. RM proximal to $F C u$. An scarcely reaching $\mathrm{FCu}$. Anal lobe not developed. Squama bare. Arculus pale, brachiolum pale with 1 seta. Legs: All segments uniformly yellowish dark brown. Fore tibia with a short apical spur; mid and hind tibial combs slightly separated each other, each with a spur. LR 1.60. Pulvillus absent. 
Abdomen: Uniformly pale yellow. Hypopygium (Fig. 3C): Anal tergite apically round, with 3-4 apical setae each side of anal point and 6 median setae; Anal tergite band V-type, almost reaching to base of anal point. Anal point short, flattened at tip, with unique anal crests. Superior volsella round, with 9-10 dorsal setae; digitus short, pointed at tip, only tip projecting beyond margin of superior volsella (Fig. $3 \mathrm{D})$. Median volsella moderately long $(50 \mu \mathrm{m})$, slightly curved outward at tip, with many simple setae, directed outward. Inferior volsella cylindrical, with 14-16 setae at tip. Gonocoxite short. Gonostylus tapered apically, with an apical and several inner-lateral setae distally.

Distribution. Europe (England, France, Belgium), Mongolia, Japan, Korea.

Remarks. This species was reported in Europe (Edwards, 1929; Goetghebuer, 1937; Pinder, 1978) and Japan (Sasa and Kamimura, 1987). Sasa and Kamimura (1987) remarked that specimens collected in Japan was tentatively diagnosed as Paratanytarsus inopertus, because of the different body coloration (mostly dark brown in Japanese specimens, whereas light green in England species). However, Edwards (1929) found that there was a variation form in body color. Our specimens differ from Japanese specimens, and are similar to those of Europe in body coloration. LR is 1.2 in Japanese sample, but 1.4-1.5 in English samples, and our specimens are 1.60. Therefore, our specimens coincide well with $\mathrm{Pa}$. inopertus in Europe.

\section{1*Paratanytarsus paramikesecumdus sp. nov.}

Material examined. Holotype, $\sigma^{7}$ (R-CH-7432), Korea: Jeollabuk-do, Muju-gun, Muju-eup, Dangsan-ri, 22 May 2009, Jeong KY. Paratypes, $50^{7} \sigma^{7}$ (R-CH-6502, 7315, 7364, $7404,7448)$, data same as holotype.

Diagnosis. Rather small, yellowish species (WL $1.4 \mathrm{~mm}$ ). $\mathrm{R}_{2+3}$ absent. Anal tergite with 10-12 median setae on middle. Anal point short, rounded at tip, with a scale-like ridges. Superior volsella round, with 2 inner-marginal setae and 1 dorsal seta; digitus slightly pointed, long projecting beyond margin of superior volsella. Median volsella short, with 5-6 spoon-shaped setae and several simple setae. AR 0.95. LR 1.70 .

Description (male). Head: Eye bare, with moderately produced dorsomedial extension. Frontal tubercle absent. 7-8 postoculars. Antenna brownish yellow, with 13 segments, AR 0.95. Palp pale, with 5 segments: 30, 36, 91, 104, 143 $\mu \mathrm{m}(1: 1.2: 3.0: 3.5: 4.8)$. Clypeus pale yellow, with 15 setae. Thorax: Yellow in ground color. Antepronotum yellowish, narrowed dorsally, widely separated. Scutum over- reaching antepronotum, light yellow, vittae absent. Scutellum yellow, with 3 setae. Postnotum yellow. Halter pale. Wing (Fig. 4A): WL $1.4 \mathrm{~mm}$. Membrane covered with macrotrichiae, mostly on distal half. Costa not produced, $\mathrm{R}_{1}$ and $\mathrm{R}_{4+5}$ running closed each other. $\mathrm{R}_{2+3}$ absent. $\mathrm{FCu}$ slightly distal to $\mathrm{RM}$. $\mathrm{Cu}_{1}$ almost straight. An just reaches $\mathrm{FCu}$. Anal lobe not developed. Squama bare. Arculus pale, brachiolum pale, with 1 seta. All veins, except subcosta and M, setosed. Legs: All segments uniformly pale yellow. Fore tibia with a weak spur apically; mid and hind tibial combs narrowly separated, each with a short spur. Pulvillus absent. LR 1.70. Abdomen: Uniformly pale yellow. Hypopygium (Fig. 4B): Anal tergite with 10-12 median setae and 2-3 apical setae each side of anal point. Anal point short, rounded at tip, with a pair of scale-like ridges. Superior volsella round, with 2 innerlateral setae and 1 outer-lateral seta; digitus long, projecting beyond margin of superior volsella. Median volsella short, with spoon-shaped setae. Inferior volsella cylindrical, slightly bulbous at tip, with 7-9 comparatively short setae. Gonostylus tapered apically, with 10-12 short setae along inner margin, arised irregularly.

Etymology. The new species name refers to Pa. mikesecundus which is very similar in morphology.

Distribution. Korea (Muju).

Remarks. This species is very close to Paratanytarsus mikesecundus Sasa in most morphological characters, but differs in several key characters: (1) the wing membrane with macrotrichiae mostly on the distal half, whereas the wing membrane of the later species is covered with hairs much more thickly including the basal portion; (2) the anal tergite of $P a$. mikesecundus has a conical tubercle on posterior margin; (3) the frontal tubercle is absent, whereas it is present in the latter; and (4) the median volsella has 5-6 spoon-shaped setae in the former, whereas it has numerous spoon shaped setae in the latter species (Sasa, 1985).

\section{Genus Rheotanytarsus Bause, 1914}

\section{${ }^{2 *}$ Rheotanytarsus parapentapodus $\mathbf{s p . ~ n o v . ~}$}

Material examined. Holotype, $\sigma^{7}$ (R-CH-6429), Korea: Jeollabuk-do, Muju-gun, Muju-eup, Dangsan-ri, 28 Aug 2009, Jeong KY. Paratypes, 6 ช శூ $^{7}$ (R-CH-6420, 6421, 6455, $6465,6974,7143)$, same as holotype.

Diagnosis. Small, pale yellow species (WL $1.4 \mathrm{~mm}$ ). Superior volsella round, with 3 dorsal setae; digitus absent. Median volsella short, abruptly curbed at tip, directed inward, with both lamellated and simple setae. Gonostylus abruptly narrowed and curbed apically. AR 0.86. LR 2.80.

Korean name: ${ }^{1 *}$ 고사리옆장부깔따구, ${ }^{2 *}$ 집 게 유장부깔따구 

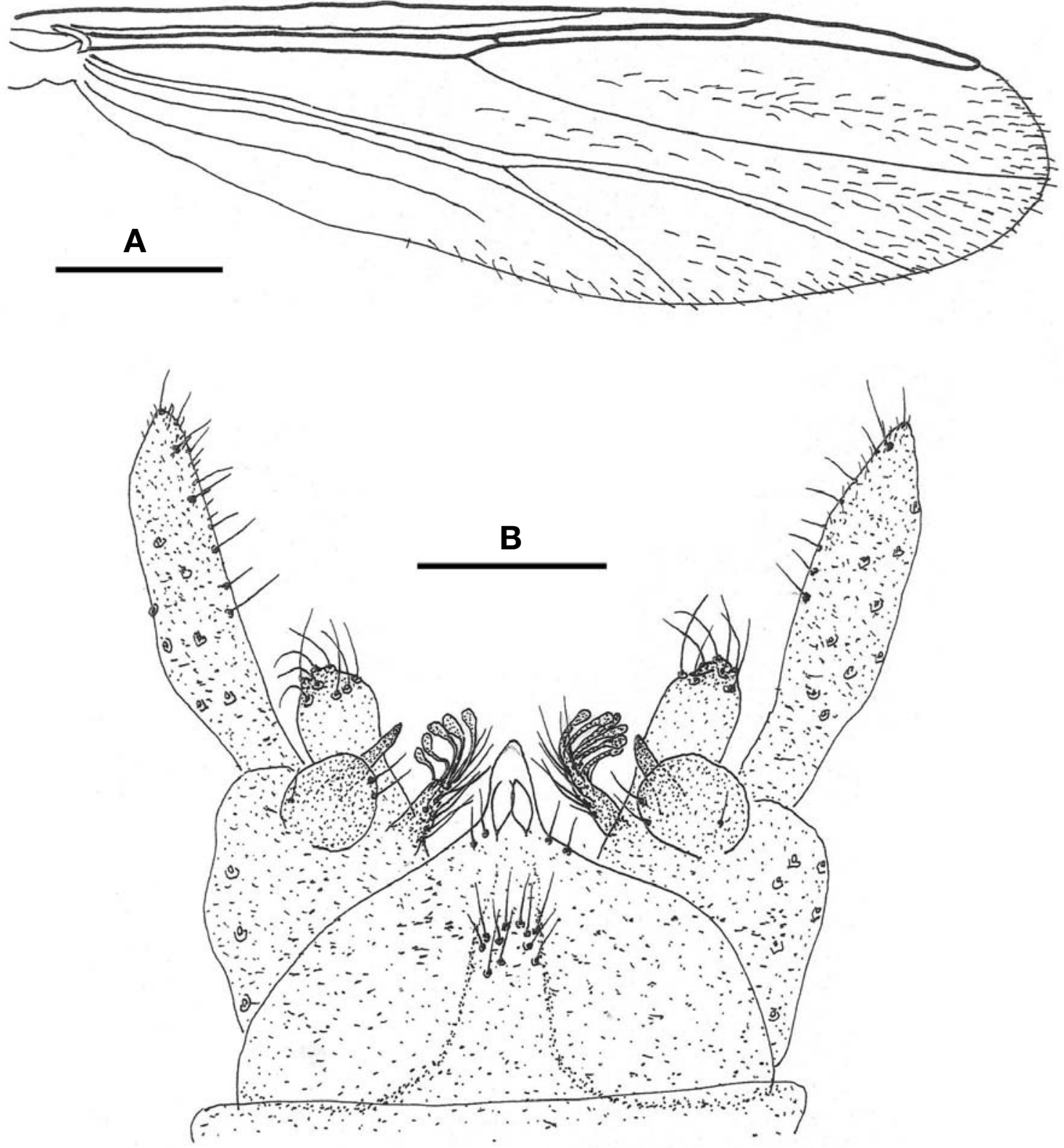

Fig. 4. Paratanytarsus paramikesecundus (male). A, Wing; $B$, Hypopygium. Scale bars: $A=0.2 \mathrm{~mm}, B=0.05 \mathrm{~mm}$.

Description (male). Head: Pale yellow. Eye bare, dorsomedially produced. Frontal tubercle absent. Antenna pale, yellowish brown, with 13 segments. AR 0.86. Palp pale yellow, with 5 segments: $36,36,121,130,211 \mu \mathrm{m}(1: 1$ : $3.4: 3.6: 5.9)$. Clypeus pale yellow, with 15 setae. Thorax: Pale yellow in ground color. Antepronotum pale yellow, narrowed dorsally, widely separated. Scutum pale yellow, vittae absent, 16 acrosticals, 7-8 dorsocentrals and 1 prealar each side. Scutellum pale, with 7 setae. Postnotum pale yellow. Halter pale. Wing (Fig. 5A): WL $1.4 \mathrm{~mm}$. Wing membrane with macrotrichiae all over, denser distally. Costa not pro- duced. $\mathrm{R}_{4+5}$ lying very close to $\mathrm{R}_{1} \cdot \mathrm{R}_{2+3}$ absent. $\mathrm{RM}$ well proximal to $\mathrm{FCu}$ Anal lobe not developed. Brachiolum pale; arculus yellowish brown. All veins setosed. Squama bare. Legs: All segments pale yellow. Fore tibia with a pale slender spur apically; mid and hind tibiae with separated combs, each with a spur. Pulvillus absent. LR 2.80. Abdomen: Pale yellow. Hypopygium (Fig. 5B): Anal tergite round distally, with several apical setae; anal tergal band poorly developed. Anal point rather long, slender, with well developed anal crests. Superior volsella round, with 3 setae dorsally; digitus absent. Median volsella short, abruptly curved at tip, direct- 

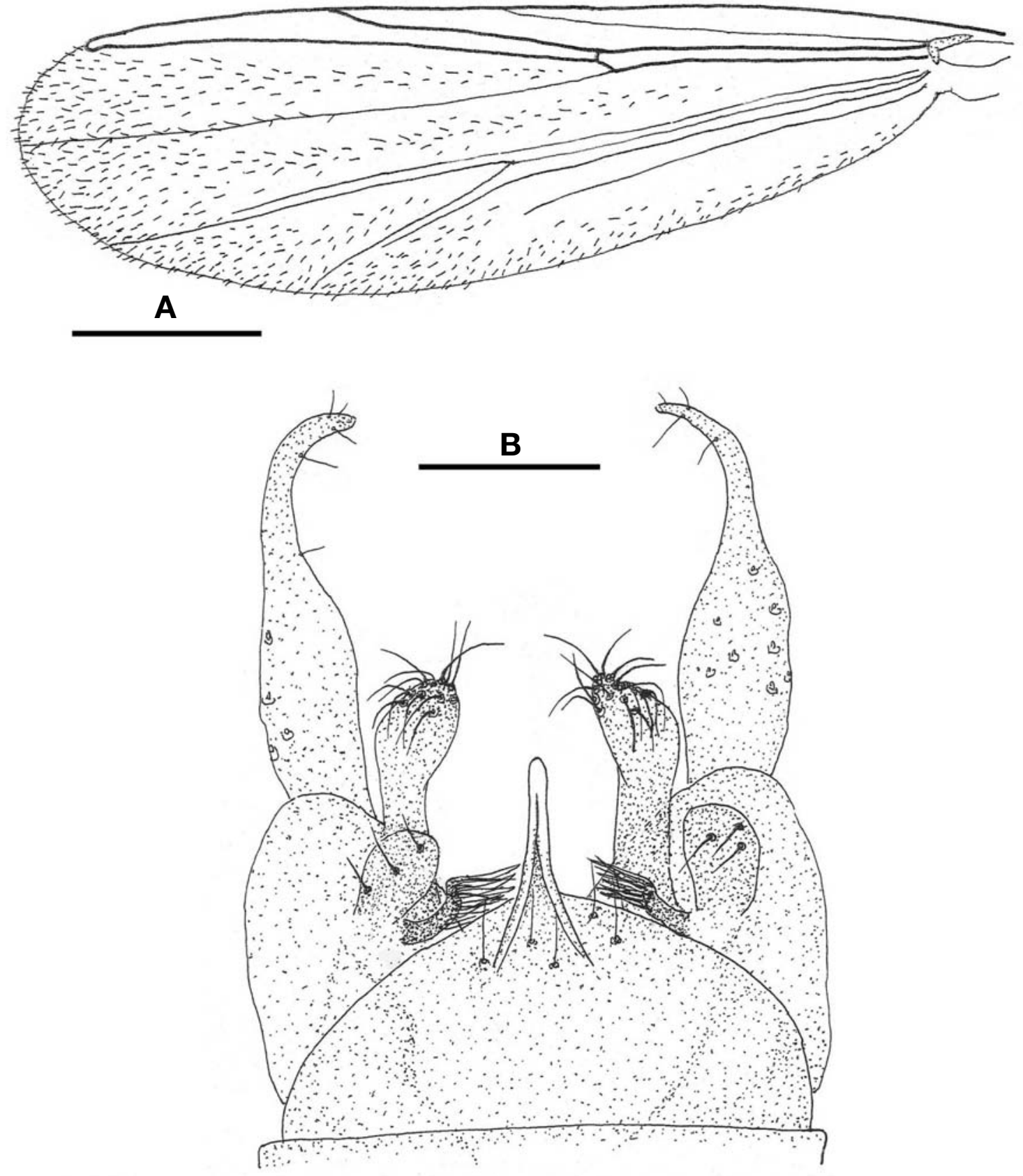

Fig. 5. Rheotanytarsus parapentapodus sp. nov. (male). A, Wing; B, Hypopygium. Scale bars: $A=0.3 \mathrm{~mm}, \mathrm{~B}=0.05 \mathrm{~mm}$.

ed inward, with both lamellar and single setae at tip. Inferior volsella rather long, cylindrical, with 14-17 setae. Gonostylus abruptly narrowed in distal $1 / 2$, bent inward apically. Etymology. The new species is named after $R h$. Pentapo$d u s$, morphologically very similar species.

Distribution. Korea (Muju).

Remarks. This new species is very similar to Rheotanytar- sus pentapodus (Kieffer), but differs in several key characters: (1) the wing length is smaller (1.4 mm vs $2.0-2.2 \mathrm{~mm})$, (2) the length of the median volsella is short, whereas that of the latter is long, extending at least to tip of superior volsella, and (3) presence of 3 dorsal setae of superior volsella, in contrast to 6-7 dorsal setae in pentapodus (Tokunaga, 1938; Pinder, 1978). 


\section{${ }^{1 *}$ Rheotanytarsus sungili sp. nov.}

Material examined. Holotype, 1 శ (R-CH-7396), Korea: Jeollabuk-do, Muju-gun, Muju-eup, Dangsan-ri, 22 May 2009, Jeong KY.

Diagnosis. Small to medium, pale yellow midges (WL 1.65 $\mathrm{mm}) . \mathrm{R}_{2+3}$ absent. Abdominal tergite pale, with dark, narrow apical band. Anal point moderately long, slightly constricted at middle, with circular anal crest (doughnut form) at base of anal point. Superior volsella ovoid; digitus absent. Median volsella short, with lamellar and simple setae. AR 0.94. LR 2.09.

Description (male). Head: Eye bar, with well developed dorsomedial extension. Frontal tubercle absent. 6-8 postoculars each side. Antenna yellowish brown, with 13 segments; AR 0.94. Palp pale yellow, with 5 segments: 32, 36, 118, $118,189 \mu \mathrm{m}(1: 1.1: 3.7: 3.7: 5.9)$. Clypeus pale yellow, with 18 setae. Thorax: Greenish pale yellow in ground color. Antepronotum pale yellow, narrowed dorsally, widely separated. Scutum overreaching antepronotum, with vandike brown vittae; 16 acrosticals, 8 dorsocentrals and 1 prealar each side. Scutellum pale yellow, with 7 setae. Postnotum vandike brown. Wing (Fig. 6A): WL $1.65 \mathrm{~mm}$. Membrane with macrotrichiae, more densely on distal $1 / 3$. Costa not extended. $R_{4+5}$ running very close to $R_{1} \cdot R_{2+3}$ absent. End of $\mathrm{R}_{4+5}$ distal to end of $\mathrm{M}_{3+4}$. $\mathrm{RM}$ well proximal to $\mathrm{FCu}$. An scarcely reaching FCu. Anal lobe not developed. Squama bare. Arculus dark brown; brachiolum pale brown, with 1 seta. Legs: All femora pale yellow, with dark brown apical end. Tibia and tarsi of foreleg pale yellowish brown; tibia and tarsi of mid and hind legs pale yellow. Fore tibia with an apical spur. Pulvillus absent. LR 2.09. Abdomen: Tergite I-VI pale, with dark apical band (Fig. 6C); tergite VIIVIII pale, without dark band. Hypopygium (Fig. 6B): Anal tergite moderately produced apically; anal tergal band almost horizontal, widely separated; coxapodeme, phallapodeme, transverse sternapodeme well developed. Anal point moderately long, slightly constricted at middle, tip smoothly rounded, with circular anal crest (doughnut-like) at the base of anal point. Superior volsella ovoid, with 5-6 dorsal setae; digitus absent. Median volsella very short, with both lamellar and simple setae. Inferior volsella cylindrical, with 1518 setae apically. Gonocoxite comparatively large. Gonostylus smoothly tapered distally, with many irregularly arranged setae on distal half.

Etymology. The new species is named after Mr. Sungil Jeong who was a police officer at Muju before his death in 2007.

Distribution. Korea (Muju).

Remarks. A unique character of this species is the circular (doughnut form) crest of the anal point. Only Rheotanytarsus tamaquintus Sasa has a similar shaped anal crest. The difference between these two species are (1) AR (0.94 vs. $0.42)$, (2) a long ventral seta of superior volsella in tamaquintus (absent in the present species), (3) a scutal vittae (distinct vs. indistinguishable), and (4) an abdominal tergite with apical band in this new species, whereas no such bands are present in tamaquintus (Sasa, 1980).

Genus Tanytarsus v.d. Wulp, 1874

${ }^{2 *}$ Tanytarsus neotamaoctavus sp. nov.

Material examined. Holotype, ס ${ }^{7}$ (CH-6758), Korea: Jeollabuk-do, Muju-gun, Muju-eup, Dangsan-ri, 22 May 2009, Jeong KY. Paratypes, 12 万 ד (CH-6701, 6763, 6811, 6817, $6828,6835,6857,6861,6880,6881,6905,7354)$, data same as holotype; $4 \sigma^{7}$ Ћ (CH-7301, 7311, 7313, 7321), locality same, 8 Aug 2009, Jeong KY.

Diagnosis. Medium to small, yellowish midge (WL $1.5 \mathrm{~mm}$ ). $\mathrm{R}_{1}$ and $\mathrm{R}_{4+5}$ running close, and $\mathrm{R}_{2+3}$ absent. Anal point smoothly tapered apically, with well defined crest and 7-8 spinules. Superior volsella round, with upwardly twisted apical lobe; digitus absent. Gonostylus narrow. AR 0.82. LR 2.51 .

Description (male). Head: Yellowish. Eye bare, slightly produced dorsomedially. Frontal tubercle absent. 8-10 postoculars. Antenna pale brown, with 13 segments. AR 0.82. Clypeus pale yellow, with 13 setae. Palp pale, with 5 segments: 31, 31, 112, 110, $153 \mu \mathrm{m}(1: 1: 3.7: 3.6: 5.0)$. Thorax: Deep yellow in ground color. Antepronotum light yellow, lobes separated medially. Scutum overreaching antepronotum, brownish yellow vittae not clearly defined. Scutellum pale, with 3 setae. Postnotum brownish yellow. Halter pale. Wing (Fig. 7A): WL $1.5 \mathrm{~mm}$. Membrane covered with macrotrichiae mostly on the distal half. All veins setosed, except subcosta and $\mathrm{R} . \mathrm{R}_{1}$ and $\mathrm{R}_{4+5}$ running closed. $\mathrm{R}_{2+3}$ absent. $\mathrm{RM}$ proximal to $\mathrm{FCu}$. $\mathrm{Cu}_{1}$ almost straight. Anal lobe not developed. Squama bare. Arculus dark brown, brachiolum pale, with 1 seta. Legs: Uniformly pale yellow. Fore tibia with an apical spur; mid and hind tibial combs widely separated, each with a spur (Fig. 7C). Pulvillus not developed. LR 2.51. Abdomen: Uniformly pale yellow. Hypopygium (Fig. 7B): Anal tergite pale yellow, weakly developed and tergite band widely separated, with 3-4 short median setae and 2-3 apical setae each side of anal point. Anal point smoothly tapered apically, with round apex, and with well defined anal crest and 7-8 groups of spines. Superior volsella round, with upwardly twisted apical lobe, with 3 inner-

Korean name: ${ }^{1 *}$ 성일유장부깔따구, ${ }^{2 *}$ 꼬부랑장부깔따구 

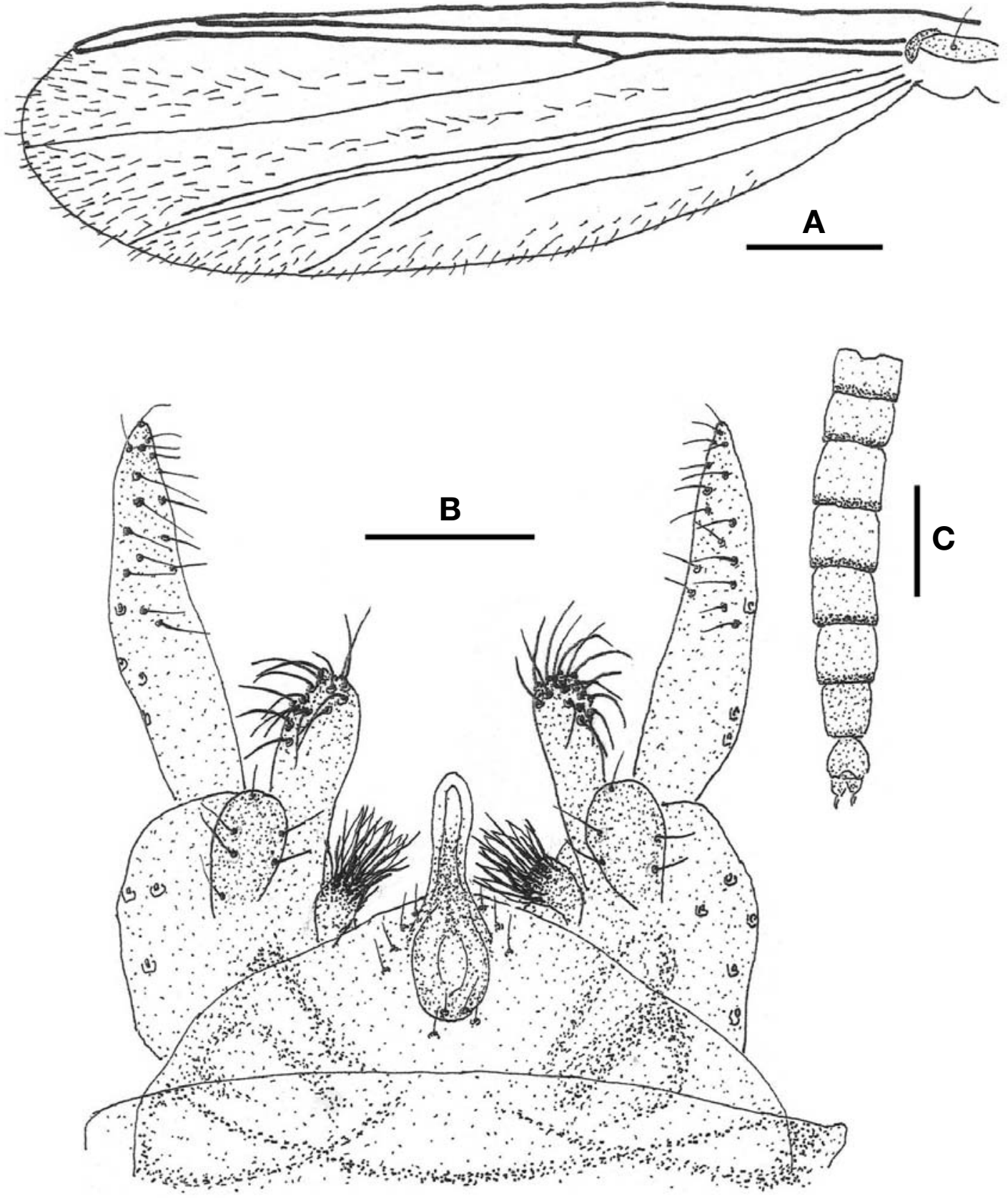

Fig. 6. Rheotanytarsus sungili sp. nov. (male). A, Wing; B, Hypopygium; C, Abdomen (dorsal). Scale bars: $A=0.1 \mathrm{~mm}, \mathrm{~B}=0.05 \mathrm{~mm}$, $\mathrm{C}=0.5 \mathrm{~mm}$.

lateral and 4-5 dorsal setae. Digitus absent. Median volsella absent. Inferior volsella cylindrical, slightly clubbed at tip, with 9-11 rather short setae. Gonostylus narrow, with an apical and 4-5 inner-lateral short setae.

Etymology. This species name refers to closely related species Ta. tamaoctavus.

Distribution. Korea (Muju).

Remarks. This new species is extremely similar to Tanytar- sus tamaoctavus Sasa in most morphological characters, including body colors, twisted digitus, and absence of median volsella. The differences are (1) anal point of our new species is rather wide (not slender), not expanded at middle and slightly tapered apically, with clear anal crest and 7-8 spine clusters, whereas that of Ta. tamaoctavus is slender, roughly parallel-sided, slightly expanded in the middle, with 3-4 spine clusters, (2) the value of AR is 0.82 in the former 

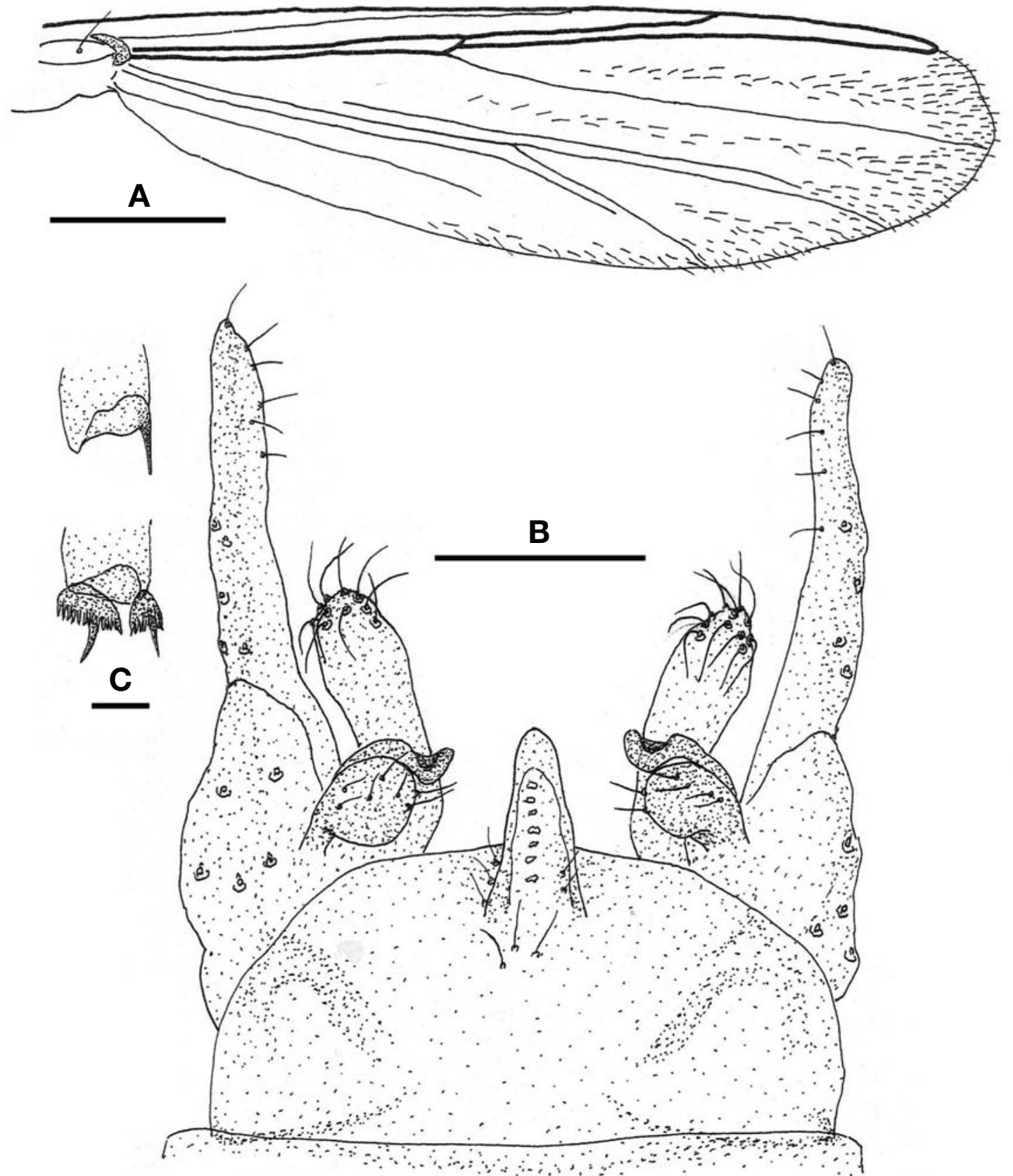

Fig. 7. Tanytarsus neotamaoctavus sp. nov. (male). A, Wing; B, Hypopygium; C, Fore tibial spur (above) and hind tibial combs (below). Scale bars: $A=0.3 \mathrm{~mm}, B=0.05 \mathrm{~mm}, C=0.01 \mathrm{~mm}$.

species and 0.65 in the latter, (3) the gonostylus is more slender in the former, and (4) $\mathrm{R}_{2+3}$ is absent in our specimens (Sasa, 1980). Na (2004) collected 10 specimens of Ta. tamaoctavus at Gapyeong, Gyeonggi-do, Korea; her description and illustration coincide well with those of Ta. tamaoctavus.

\section{${ }^{1 *}$ Tanytarsus synyunosecundus sp. nov.}

Material examined. Holotype, $\sigma^{7}$ (R-CH-6716), Korea: Jeollabuk-do, Muju-gun, Muju-eup, Dangsan-ri, 5 Sep 2008, Jeong KY, Nam SH. Paratypes, 8 『 ర ণ (R-CH-6447, 6611, $6619,6630,6749,6829,6882,6883)$, locality same, 22 May 

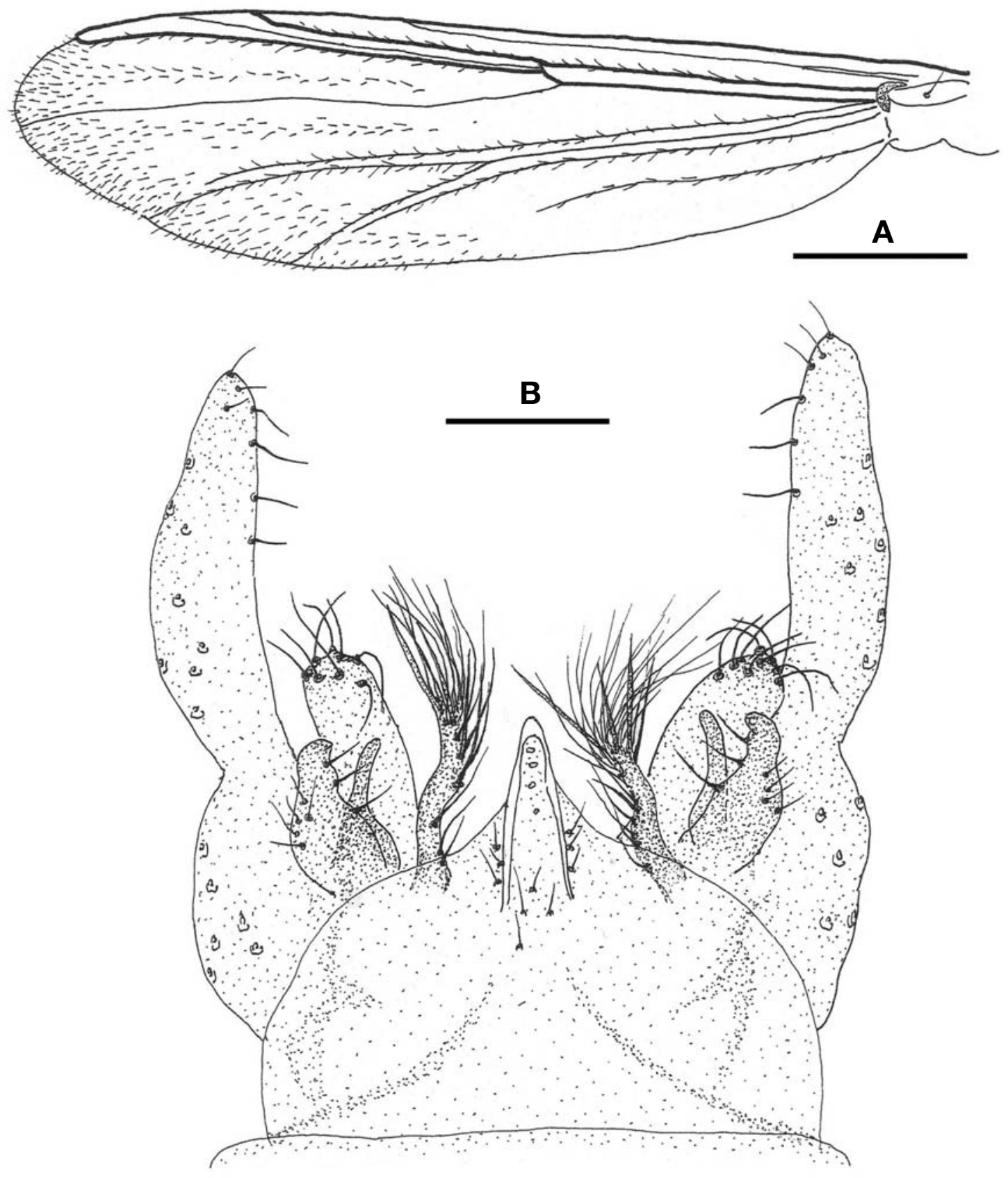

Fig. 8. Tanytarsus synyunosecundus sp. nov. (male). A, Wing; $B$, Hypopygium. Scale bars: $A=0.3 \mathrm{~mm}, B=0.05 \mathrm{~mm}$.

2009, Jeong KY; 4 ð ठ (R-CH-6953, 7000, 7051, 7237), locality same, 28 Aug 2009, Jeong KY.

Diagnosis. Medium to small, pale yellow midge (WL 1.4 $\mathrm{mm}$ ). Anal point largely overlying anal tergite, smoothly tapered apically, with well defined crest and 4-5 spine clusters. Superior volsella oval, elongated apically; digitus long, finger-like, projecting well beyond margin of superior volsella. Median volsella long, slightly curved, with many long, simple setae. AR 1.07. LR 3.16.

Description (male). Head: Eye bare, with short dorsomedial projection. Frontal tubercle moderately developed (18 $\mu \mathrm{m}$ long $\times 15 \mu \mathrm{m}$ wide). Antenna yellowish brown, with 13 segments. AR 1.07. Palp pale, with 5 segments: 32, 6, 114, $107,179 \mu \mathrm{m}(1: 1.1: 3.6: 3.4: 5.6)$. Clypeus pale yellow, with 15 setae. Thorax: Uniformly pale yellow. Antepronotum wide, dorsomedially separated. Scutum without vittae; 8 
dorsocentrals and 1 prealar each side. Scutellum pale, with 5 setae. Postnotum pale yellow. Halter pale. Wing (Fig. 8A): WL $1.4 \mathrm{~mm}$. Membrane with macrotrichiae on distal half. All veins setose, except $R$. Costa not produced. $R_{2+3}$ weakly developed, ending at middle between apices of $R_{1}$ and $R_{4+5}$. $\mathrm{RM}$ proximal to $\mathrm{FCu}$. $\mathrm{Cu}_{1}$ almost straight. Anal lobe not developed. Squama bare. Arculus dark brown, brachiolum pale, with 1 seta. Legs: Uniformly pale yellow. Fore tibia with an apical spur; mid and hind tibial combs narrowly separated, each with a spur. Pulvillus absent. LR 3.16. Abdomen: All segments pale yellow. Hypopygium (Fig. 8B): Anal tergite roundly produced distally; anal tergite band poorly developed, widely separated each other; 4-5 minute median setae. Anal point largely overlying anal tergite, smoothly tapered apically; tip round, with strong crest and 4-5 spines. Superior volsella oval, elongated apically, with 3 inner-lateral and 4-5 dorso-lateral setae; digitus fingerlike, projecting well beyond margin of superior volsella. Median volsella very long, with numerous long, simple setae. Inferior volsella cylindrical, with 8-9 apical setae. Gonostylus rather long, slightly curved at base, fused with gonocoxite, with an apical and 4-5 inner-lateral setae.

Etymology. The name synyunosecundus is given because this new species is very close to Ta. yunosecundus.

Distribution. Korea (Muju).

Remarks. Most characters of this new species coincided well with those of Tanytarsus yunosecundus Sasa, including the hypopygium, but it differs in that (1) the wing length is much shorter (1.4 mm vs. $2.1 \mathrm{~mm}$ ), (2) the value of LR is significantly larger (4.16 vs. 2.40), (3) the frontal tubercle is wider and shorter $(18 \mu \mathrm{m}$ long $\times 15 \mu \mathrm{m}$ wide vs $24 \mu \mathrm{m}$ long $\times 12 \mu \mathrm{m}$ wide), and (4) the pulvillus is absent, whereas a moderately developed pulvillus is present in Ta. yunosecundus (Sasa, 1984). Body size (represented by wing length) and size of frontal tubercles show considerable range of variation according to geographical and other environmental conditions. However, the LR and pulvillus belong to rather stable characteristics.

\section{${ }^{1 *}$ Tanytarsus tamagotoi Sasa, 1983}

Tanytarsus tamagotoi Sasa, 1983: 23; Sasa and Kikuchi, 1986: 3.

Material examined. $2 \sigma^{\nearrow}$, Korea: Jeollabuk-do, Mujugun, Muju-eup, Dangsan-ri, 5 Sep 2008, Jeong KY, Nam

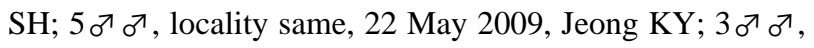
locality same, 28 Aug 2009, Jeong KY.

Diagnosis. Medium to small, yellowish midge (WL 1.88 $\mathrm{mm}$ ). Anal point abruptly widened at base, with round tip, well defined crest and 5-7 spine clusters. Superior volsella ovoid, with small apical lobe, and 3 inner-lateral and 4-5 outer-lateral setae dorsally, and 1 ventral seta arising from cylindrical base; digitus long, finger-like. Median volsella short, with numerous simple setae. Gonostylus fused with gonocoxite. AR 1.16. LR 2.61.

Description (male). Head: Eye bare, slightly produced dorsomedially. 10-12 postoculars. Frontal tubercle absent. Antenna dark brown, with 13 segments, AR 1.16. Palp pale, with 5 segments: $36,36,107,118,143 \mu \mathrm{m}(1: 1: 3.0: 3.3: 4.0)$. Clypeus pale yellow, with 12 setae. Thorax: Yellow in ground color. Antepronotum yellow, dorsally narrowed, separated dorsomedially. Scutum overreaching antepronotum, vittae absent. Scutellum pale yellow, with 3 setae. Postnotum yellow. Wing (Fig. 9A): WL $1.88 \mathrm{~mm}$. Membrane with macrotrichiae, mostly on distal half. Costa not produced. $R_{2+3}$ running close to $R_{4+5}$ and ending at $1 / 3$ basal between ends of $R_{1}$ and $R_{4+5}$. $R M$ proximal to $F C u$. $\mathrm{Cu}_{1}$ almost straight. Anal lobe not developed. Squama bare. Arculus pale yellow, brachiolum pale, with 1 seta. Legs: All segments uniformly pale yellow. Foretibia with 1 spur apically; mid and hind tibial combs separated, each with 1 spur. Pulvillus absent. LR 2.61. Abdomen: Uniformly pale yellow. Hypopygium (Fig. 9B): Anal tergite smoothly rounded distally, poorly developed anal tergite band widely separated; apodeme weakly developed. Anal point widened at base, with round tip, and well developed crest and 5-7 spine clusters. Superior volsella somewhat ovoid, with a small, apical lobe curved inward at tip, with 2-3 inner-lateral and 4-5 outerlateral setae dorsally, and 1 ventral seta arising from cylindrical base; digitus long, finger-like, well projecting margin of superior volsella (Fig. 9C). Median volsella short, with numerous simple setae. Inferior volsella cylindrical, convex at base internally, with 7-9 erect setae at tip. Gonostylus fused with gonocoxite, inner margin almost straight, with an apical, several setae and numerous microtrichiae distally.

Distribution. Japan and Korea.

Remarks. Our specimens coincide well with Tanytarsus tamagotoi described by Sasa (1983) and Sasa and Kikuchi (1986) in most key characters, except for wing length (1.88 mm vs. 1.47) and AR (1.16 vs. 0.87).

\section{${ }^{2 *}$ Tanytarsus uresiacutus Sasa, 1989}

Tanytarsus uresiacutus Sasa, 1989: 79.

Material examined. 6 ऽ gun, Muju-eup, Dangsan-ri, 5 Sep 2008, Jeong KY, Nam SH; $2 \sigma^{\nearrow}$ ð , locality same, 28 Aug 2009, Jeong KY.

Diagnosis. Small, right yellow species (WL $1.2 \mathrm{~mm}$ ). Anal

Korean name: ${ }^{1 *}$ 가위장부깔따구, ${ }^{2 *}$ 즐거워장부깔따구 


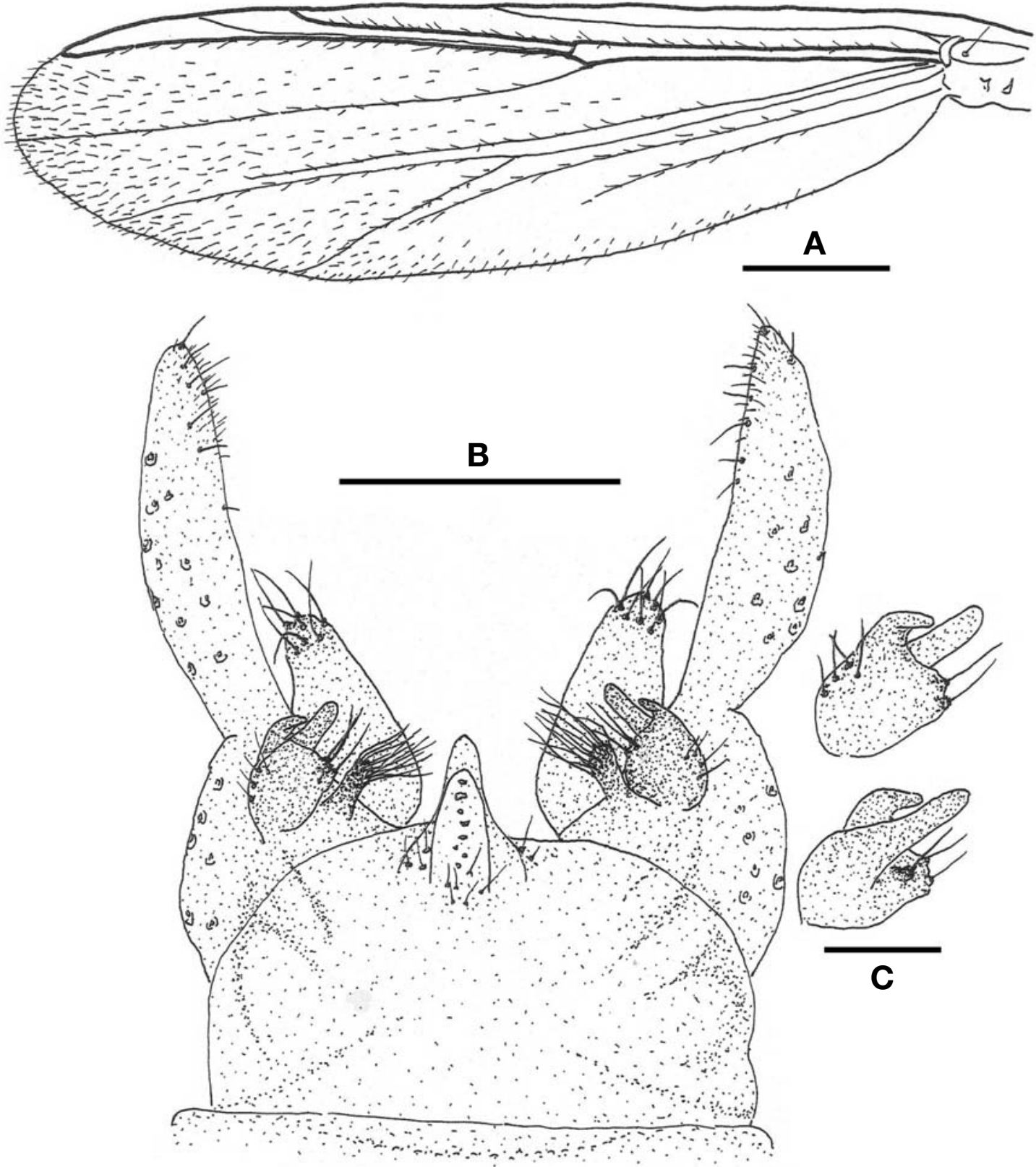

Fig. 9. Tanytarsus tamagotoi (male). A, Wing; B, Hypopygium; C, Superior volsella (dorsal and ventral from above). Scale bars: $A=0.3 \mathrm{~mm}, B=0.1 \mathrm{~mm}, C=0.03 \mathrm{~mm}$.

point rather small, tapered at tip, with distinct anal crest; anal spines absent. Superior volsella ovoid, with 4-5 minute setae dorsally; finger-like digitus projecting beyond margin of superior volsella. Median volsella short, directed backward, with 5-7 spoon-shaped lamellar setae. Gonostylus rather small, tapered apically. AR 0.73. LR 2.42.

Description (male). Head: Eye bare, dorsomedially extended. Antenna yellowish brown, with 13 segments, AR 0.73. Frontal tubercle absent. Clypeus pale yellow, with 13 setae.
Palp pale, with 5 segments: 27, 28, 77, 90, $115 \mu \mathrm{m}(1: 1.0$ : $2.9: 3.3: 4.3)$. Thorax: Light yellow in ground color. Antepronotum pale yellow, narrowed dorsally, medially separated each other. Scutum light yellow, vittae absent. Scutellum pale yellow, with 4 scutellars. Postnotum pale yellow. Wing (Fig. 10A): WL $1.2 \mathrm{~mm}$. Membrane with macrotrichiae on distal half. Costa not produced. $\mathrm{R}_{2+3}$ absent. $\mathrm{R}_{4+5}$ lying close to $\mathrm{R}_{1}$. RM well proximal to $\mathrm{FCu}$. Anal lobe not developed. Brachiolum and arculus pale. Squama bare. Legs: All legs 

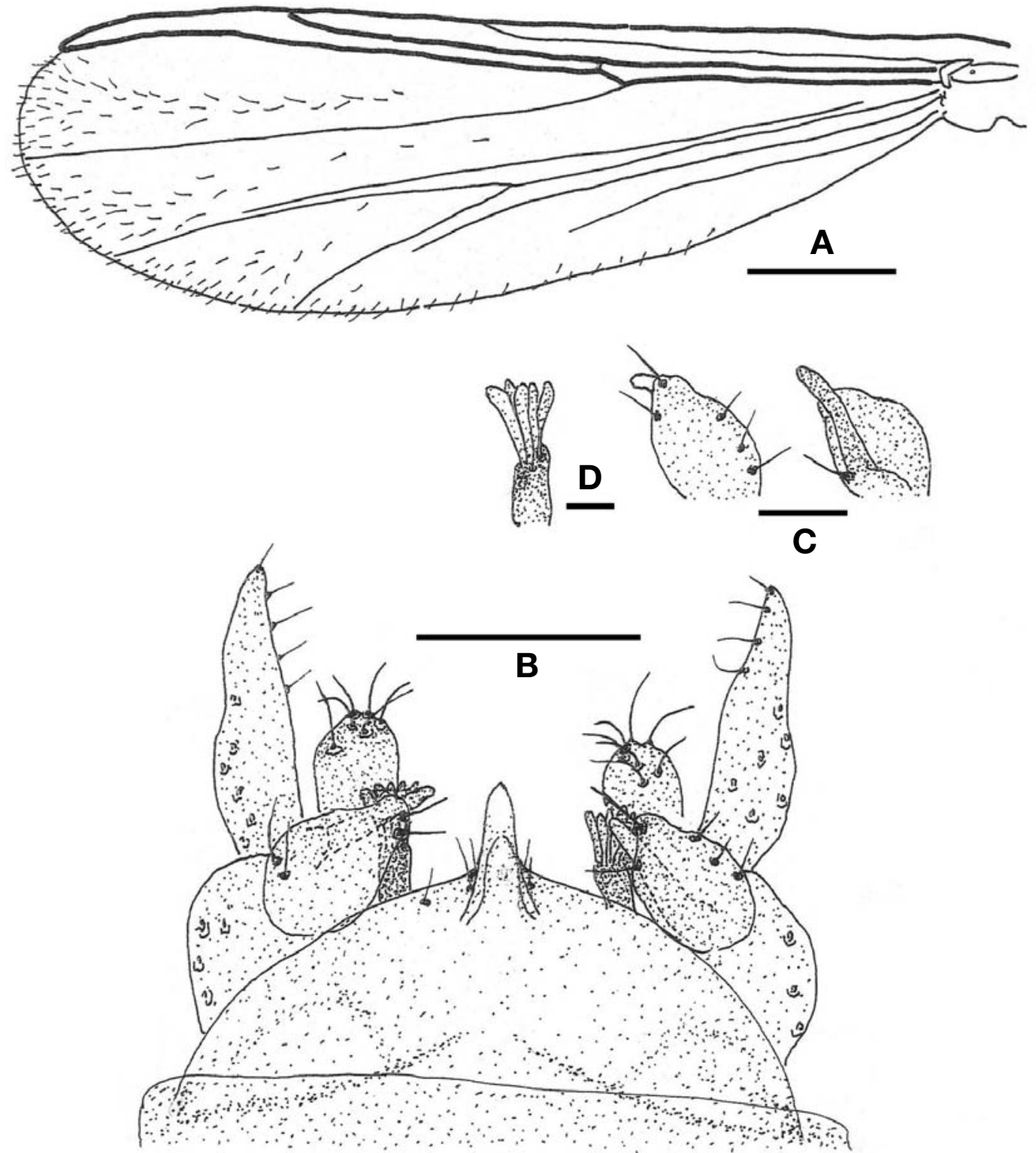

Fig. 10. Tanytarsus uresiacutus (male). A, Wing; $B$, Hypopygium; $C$, Superior volsella (dorsal and ventral from left); $D$, Median volsella. Scale bars: $A=0.5 \mathrm{~mm}, B=0.05 \mathrm{~mm}, C=0.02 \mathrm{~mm}, D=0.01 \mathrm{~mm}$.

uniformly pale yellow. Fore tibia with a short spur; mid and hind tibial combs separate, with only one spur. Puvillus not developed. LR 2.42. Abdomen: Uniformly pale yellow. Hypopygium (Fig. 10B): Anal tergite smoothly rounded apically; anal tergite band and apodeme poorly developed; median setae absent. Anal point rather short, tapered at tip, with distinct anal crest distinct; anal spines absent. Superior volsella (Fig. 10C) ovoid, with 4-5 minute setae dorsally; finger-like digitus projecting slightly beyond margin of superior volsella. Median volsella (Fig. 10D) short, directed back- ward, with 5-7 spoon-shaped lamellar settae. Inferior volsella cylindrical, with 6-8 rather short setae apically. Gonostylus rather small, tapered apiclly, with 1 apical and 5-6 innerlateral setae.

Distribution. Japan and Korea.

Remarks. Sasa (1989) described a new species, Tanytarsus uresiacutus with 2 males collected at Ureshino river in Toyama, Japan. Our specimens coincide well with $T$. uresiacutus in morphological key characters, except the absence of frontal tubercles. He described that moderate size $(22 \mu \mathrm{m}$ 
long) of frontal tubercles are present. Two males of Sasa's specimens lacked forelegs so that there was no value for the LR. LR of our specimens is 2.42 .

\section{REFERENCES}

Edwards FW, 1929. British non-biting midges (Diptera, Chironomidae). Transactions of the Royal Entomological Society of London, 77:279-430.

Goetghebuer M, 1937. Tendipedidae (Chironomidae). b) Subfamilie Tendipedinae (Chironominae). A, Die Imagines. In: Die Fliegen der Palaearktischen Region (Ed., Lindner E), $13 \mathrm{c}: 1-138$.

$\mathrm{Na} \mathrm{KB}, 2004$. Taxonomy of Chironomidae (Diptera, Insecta) in Seoul-Gyeonggi area, Korea. MSc thesis, Seoul Women's University, Seoul, Korea, p. 100.

Pinder LCV, 1978. A key to adult males of British chironomidae. Freshwater Biological Association Scientific Publication, 37:1-169.

Ree HI, 1989. Studies on Korean Chironomidae (Diptera). IV. A new species and two unrecorded species from Korea. Korean Journal of Entomology, 19:207-214.

Ree HI, 1992. Three new species of Chironomidae (Diptera) from Korea. Korean Journal of Systematic Zoology, Special Issue (3):129-138.

Ree HI, Jeong KY, 2010. Fauna of non-biting midges (Diptera, Chironomidae) from Soyang river in Chuncheon-si, Gangwon-do, Korea. Korean Journal of Systematic Zoology, 26:115-140.

Ree HI, Kim JY, 2003. A new species of Tanytarsus and an unrecorded species of Chironomus in Korea (Diptera: Chiromidae). Korean Journal of Systematic Zoology, 19:11-17.

Ree HI, Kim MS, 1988. Studies on Korean Chironomidae (Diptera) III. Description of two unrecorded species from Korea and three new species. Korean Journal of Systematic Zoology, Special Issue 2:13-24.

Saether OA, 1980. Glossary of chironomid morphology terminology (Diptera: Chironomidae). Entomologica Scandinavica, Supplement 14:1-51.

Sasa M, 1980. Studies on chironomid midges of the Tama River. Part 2. Description of 20 species of Chironominae recovered from a tributary. Research Report from the National Institute for Environmental Studies, Japan, 13:9-107.
Sasa M, 1983. Studies on chironomid midges of the Tama River. Part 5. An observation on the distribution of chironominae along the main stream in June, with description of 15 new species. Research Report from the National Institute for Environmental Studies, Japan, 43:1-67.

Sasa M, 1984. Studies on chironomid midges in lakes of the Nikko National Park. Part 2. Taxonomical and morphological studies on the chironomid species collected from lakes in the Nikko National Park. Research Report from the National Institute for Environmental Studies, Japan, 70:19215.

Sasa M, 1985. Studies on chironomid midges of some lakes in Japan. Research Report from the National Institute for Environmental Studies, Japan, 83:25-99.

Sasa M, 1989. Studies on chironomid midges (Diptera, Chironomidae) of Shou river. Research Report from Toyoma Prefectural Environmental Pollution Research Center, 1989: 26-110.

Sasa M, Kamimura K, 1987. Chironomid midges collected on the shore of lakes in the Akan National Park, Hokkaido (Diptera, Chironomidae). Research Report from the National Institute for Environmental Studies, Japan, 104:9-61.

Sasa M, Kawai K, 1987. Studies on the chironomid midges of Lake Biwa (Diptera, Chironomidae). Lake Biwa Study Monograph, 3:1-119.

Sasa M, Kikuchi M, 1986. Notes on the chironomid midges of the subfamilies Chironominae and Orthocladiinae collected by light traps in a rice paddy area in Tokushima (Diptera, Chironomidae). Japanese Journal of Sanitary Zoology, 37:1739.

Shcherbina GKh, Zelentsov NI, 2008. Chironomids fauna (Diptera, Chironomidae) in some waterbodies and watercourses of Mongolia. Inland Water Biology, 1:19-24.

Tokunaga M, 1938. Chironomidae from Japan (Diptera), X: new of little-known midges, with descriptions of the metamorphoses of several species. Phillipine Journal of Science, 65:314383.

Walker F, 1856. Insecta Britannica. Diptera, 3:1-35.

Wang X, Zheng L, 1992. Checklist of Chironomidae records from China. Netherlands Journal of Aquatic Ecology, 26: 247-255. 